\title{
Control of Nonprehensile Rolling Manipulation: Balancing a Disk on a Disk
}

\author{
Ji-Chul Ryu, Fabio Ruggiero, and Kevin M. Lynch
}

\begin{abstract}
This paper presents stabilization control of a rolling manipulation system called the disk-on-disk. The system consists of two disks in which the upper disk (object) is free to roll on the lower disk (hand) under the influence of gravity. The goal is to stabilize the object at the unstable upright position directly above the hand. We use backstepping to derive a control law yielding global asymptotic stability. We present simulation as well as experimental results demonstrating the controller.
\end{abstract}

\section{INTRODUCTION}

Nonprehensile manipulation primitives such as rolling, sliding, pushing, and throwing are commonly used by humans but are often avoided by robots, who seem to prefer grasping. Dynamic nonprehensile manipulation raises challenges in high-speed sensing and control, as the manipulated object is not in static equilibrium throughout the process. An advantage, however, is that dynamics can be exploited to help the robot control object motions that would otherwise be impossible [1], [2].

Our long-term goal is to develop a unified framework for planning and control of dynamic robotic manipulation. A typical manipulation plan consists of a sequence of manipulation primitives chosen from a library of primitives, with each primitive equipped with its own feedback controller. The primitive under study in this paper is nonprehensile rolling manipulation, where a single object rolls on the surface of a controlled manipulator. Problems of interest include planning the motion of the manipulator to achieve the desired rolling motion of the object (e.g., [2], [3]) and feedback control to stabilize the desired trajectory.

In this paper we study feedback stabilization of a canonical rolling example: balancing a disk-shaped object on top of a disk-shaped manipulator in a vertical plane. We constrain the motion of the manipulator to rotation about its center. Using backstepping and assuming rolling contact at all times, we derive a control law that asymptotically stabilizes the object to the balanced position from any initial state. The basin of attraction is necessarily reduced, but still large, when the contact is modeled according to Coulomb friction. We conclude by demonstrating a successful implementation of the rolling controller using high-speed vision feedback.

Several examples of control of rolling have been studied in the literature. Among them is the ball and beam system in which a ball rolls with one degree of freedom along a

J. Ryu and K. M. Lynch are with the Department of Mechanical Engineering, Northwestern University, Evanston, IL, 60208, USA. K. M. Lynch is also affiliated with the Northwestern Institute on Complex Systems. email: \{jcryu, kmlynch\}enorthwestern.edu. Fabio Ruggiero is with the Dipartimento di Informatica e Sistemistica, Università degli Studi di Napoli Federico II, Naples, Italy, fabio.ruggiero@unina.it. linear beam. The beam rotates in a vertical plane and the ball rolls under gravity. To solve the stabilization problem, approximate input-output linearization is used in [4]. In [5], global asymptotic stability is achieved by a saturation control law which uses state-dependent saturation levels. A related problem is balancing a ball on a plate. In [6], a gimbalsuspended plate is actuated by two independent motors to control the position of a ball rolling on the plate. Another type of ball and plate system is discussed in [7], where a ball captured between two horizontal plates rolls in response to the motion of one of the plates. The ball's configuration evolves according to the rolling kinematics (no dynamics).

For multi-fingered manipulation in [8], the rolling constraint is exploited in order to control both the pose of the manipulated object and the paths of contact points along the object's surface. The roles of the shape and motion of the manipulator on the motion of the rolling object were studied in the context of the "butterfly" contact juggling trick in [3]. No analysis is provided of feedback stabilization of rolling trajectories or balanced configurations, however.

In Section II, the kinematic and dynamic equations of the disk-on-disk system are derived. In Section III, a globally asymptotically stabilizing controller is designed based on backstepping. Simulation and experimental results are provided in Sections IV and V, respectively.

\section{DynAmics OF THE DISK-ON-Disk}

In this section, we derive the kinematic and dynamic equations of a smooth planar object rolling on a smooth, motion-controlled planar manipulator, referred to as the "hand." We then restrict to the special case of the disk-ondisk in Section II-C.

We define a coordinate frame $u_{\mathrm{h}}-v_{\mathrm{h}}$ attached to the hand and let $x_{\mathrm{h}} \in \mathbb{R}^{2}$ be the position and $\theta_{\mathrm{h}}$ be the orientation of the frame in a world reference frame $x-y$ (Fig. 1). The position and orientation of the frame $u_{0}-v_{0}$ attached to the center of mass of the object are given by $x_{\mathrm{o}} \in \mathbb{R}^{2}$ and $\theta_{\mathrm{o}}$, respectively.

The curve of the hand is parameterized by an arclength parameter $s_{\mathrm{h}} \in \mathbb{R}$, and the shape of the hand is given by $c_{\mathrm{h}}\left(s_{\mathrm{h}}\right) \in \mathbb{R}^{2}$ in the $u_{\mathrm{h}}-v_{\mathrm{h}}$ frame. The parameter $s_{\mathrm{h}}$ increases counterclockwise along the hand. The object is parameterized by the arclength parameter $s_{0}$, where $s_{0}$ increases clockwise, and the object shape in the $u_{0}-v_{0}$ frame is given by $c_{\mathrm{o}}\left(s_{\mathrm{o}}\right)$. With this choice, the pure rolling assumption yields $\dot{s}_{\mathrm{h}}=\dot{s}_{\mathrm{o}}$. By an appropriate choice of the location of $s_{\mathrm{o}}=0$, we have $s_{\mathrm{h}}=s_{\mathrm{o}}$ at all times during rolling. For this reason, we do not refer to $s_{\mathrm{o}}$ during rolling. 


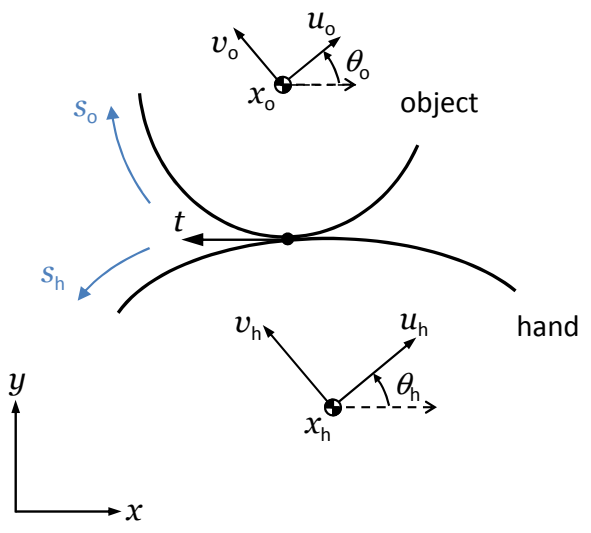

Fig. 1. A schematic of a general planar rolling manipulation system. The $u_{\mathrm{h}}-v_{\mathrm{h}}$ frame is attached to the hand and $\theta_{\mathrm{h}}$ denotes its orientation with respect to the $x-y$ world frame. Similarly, the $u_{0}-v_{0}$ frame is attached to the object and $\theta_{\mathrm{o}}$ denotes the frame's orientation.

Assuming the two disks maintain rolling contact at all times, the system's configuration is fully specified by $q=$ $\left[x_{\mathrm{h}}^{T}, \theta_{\mathrm{h}}, s_{\mathrm{h}}\right]^{T}$.

\section{A. Kinematic Equations}

As the whole configuration of the system can be described by $q=\left[x_{\mathrm{h}}^{T}, \theta_{\mathrm{h}}, s_{\mathrm{h}}\right]^{T}$, in this subsection we express the object's position $x_{\mathrm{o}}$ and orientation $\theta_{\mathrm{o}}$, and their derivatives, in terms of $q$ and its derivatives.

At a contact point $c_{\mathrm{h}}\left(s_{\mathrm{h}}\right)$, the tangent vector is expressed as $t=c_{\mathrm{h}}^{\prime}=\left[u_{\mathrm{h}}^{\prime}, v_{\mathrm{h}}^{\prime}\right]^{T}$ at an angle $\phi=\operatorname{atan} 2\left(v_{\mathrm{h}}^{\prime}, u_{\mathrm{h}}^{\prime}\right)$ in the $u_{\mathrm{h}}-v_{\mathrm{h}}$ frame, where the symbol / indicates a derivative with respect to the parameter. Using the property $\left\|\frac{d c_{\mathrm{h}}}{d s_{\mathrm{h}}}\right\|=1$ of arclength parameterizations, the signed curvature of the hand can be written as

$$
\kappa_{\mathrm{h}}\left(s_{\mathrm{h}}\right)=\frac{d \phi_{\mathrm{h}}}{d s_{\mathrm{h}}}=u_{\mathrm{h}}^{\prime}\left(s_{\mathrm{h}}\right) v_{\mathrm{h}}^{\prime \prime}\left(s_{\mathrm{h}}\right)-u_{\mathrm{h}}^{\prime \prime}\left(s_{\mathrm{h}}\right) v_{\mathrm{h}}^{\prime}\left(s_{\mathrm{h}}\right),
$$

where $\kappa_{\mathrm{h}}>0$ means the hand is convex at the contact point. Similarly, the signed curvature of the object is given by

$$
\kappa_{\mathrm{o}}\left(s_{\mathrm{h}}\right)=\frac{d \phi_{\mathrm{o}}}{d s_{\mathrm{h}}}=u_{\mathrm{o}}^{\prime}\left(s_{\mathrm{h}}\right) v_{\mathrm{o}}^{\prime \prime}\left(s_{\mathrm{h}}\right)-u_{\mathrm{o}}^{\prime \prime}\left(s_{\mathrm{h}}\right) v_{\mathrm{o}}^{\prime}\left(s_{\mathrm{h}}\right)
$$

Due to the opposite sense of the parameterization, $\kappa_{\mathrm{o}}<0$ indicates convexity. The relative curvature at $s_{\mathrm{h}}$ is

$$
\kappa_{\mathrm{r}}\left(s_{\mathrm{h}}\right)=\kappa_{\mathrm{h}}\left(s_{\mathrm{h}}\right)-\kappa_{\mathrm{o}}\left(s_{\mathrm{h}}\right),
$$

where $\kappa_{\mathrm{r}}>0$ is required to guarantee a single contact point locally.

The angle of the tangent vector measured in the world reference frame is written as

$$
\theta_{\mathrm{h}}+\phi_{\mathrm{h}}=\theta_{\mathrm{o}}+\phi_{\mathrm{o}}
$$

yielding

$$
\begin{aligned}
& \theta_{\mathrm{o}}=\theta_{\mathrm{h}}+\operatorname{atan} 2\left(v_{\mathrm{h}}^{\prime}, u_{\mathrm{h}}^{\prime}\right)-\operatorname{atan} 2\left(v_{\mathrm{o}}^{\prime}, u_{\mathrm{o}}^{\prime}\right), \\
& \dot{\theta}_{\mathrm{o}}=\dot{\theta}_{\mathrm{h}}+\dot{s}_{\mathrm{h}} \kappa_{\mathrm{r}}\left(s_{\mathrm{h}}\right), \\
& \ddot{\theta}_{\mathrm{o}}=\ddot{\theta}_{\mathrm{h}}+\ddot{s}_{\mathrm{h}} \kappa_{\mathrm{r}}\left(s_{\mathrm{h}}\right)+\dot{s}_{\mathrm{h}}^{2} \kappa_{\mathrm{r}}^{\prime}\left(s_{\mathrm{h}}\right) .
\end{aligned}
$$

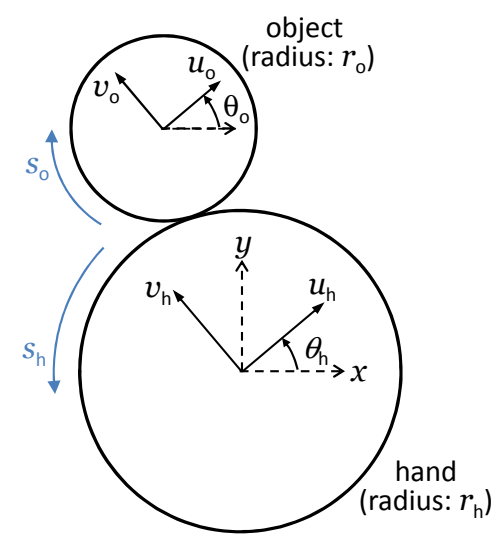

Fig. 2. A schematic of the disk-on-disk system. Both the hand and object are circular disks, and the hand is constrained to rotate about its center.

To determine the relationship of $x_{\mathrm{o}}$ and its derivatives on $q$ and its derivatives, we start with the condition that the contact point on the hand and object are coincident,

$$
x_{\mathrm{h}}+R\left(\theta_{\mathrm{h}}\right) c_{\mathrm{h}}\left(s_{\mathrm{h}}\right)=x_{\mathrm{o}}+R\left(\theta_{\mathrm{o}}\right) c_{\mathrm{o}}\left(s_{\mathrm{h}}\right),
$$

where the rotation matrix $R(\theta) \in S O(2)$ is given by

$$
R(\theta)=\left[\begin{array}{cc}
\cos \theta & -\sin \theta \\
\sin \theta & \cos \theta
\end{array}\right]
$$

Noticing that $\frac{d}{d t} R(\theta)=\dot{\theta} R\left(\theta+\frac{\pi}{2}\right)$ and plugging in (4), we obtain

$$
\begin{aligned}
x_{\mathrm{o}}= & x_{\mathrm{h}}+R\left(\theta_{\mathrm{h}}\right) c_{\mathrm{h}}-R\left(\theta_{\mathrm{o}}\right) c_{\mathrm{o}}, \\
\dot{x}_{\mathrm{o}}= & \dot{x}_{\mathrm{h}}+\left(R\left(\theta_{\mathrm{h}}+\frac{\pi}{2}\right) c_{\mathrm{h}}-R\left(\theta_{\mathrm{o}}+\frac{\pi}{2}\right) c_{\mathrm{o}}\right) \dot{\theta}_{\mathrm{h}} \\
& +\left(R\left(\theta_{\mathrm{h}}\right) c_{\mathrm{h}}^{\prime}-\kappa_{\mathrm{r}} R\left(\theta_{\mathrm{o}}+\frac{\pi}{2}\right) c_{\mathrm{o}}-R\left(\theta_{\mathrm{o}}\right) c_{\mathrm{o}}^{\prime}\right) \dot{s}_{\mathrm{h}} .
\end{aligned}
$$

The expression for $\ddot{x}_{\mathrm{o}}$ is omitted due to length.

When the relative motion between the hand and the object is of interest, the contact kinematics derived in [9] can be used if the relative curvature is invertible.

\section{B. Dynamic Equations}

The dynamic equations are derived from Lagrange's equations

$$
\frac{d}{d t} \frac{\partial L}{\partial \dot{q}}-\frac{\partial L}{\partial q}=\tau
$$

with Lagrangian $L=K-U$, where the kinetic energy $K$ and potential energy $U$ are given by

$$
\begin{aligned}
K & =\frac{1}{2}\left(m_{\mathrm{h}} \dot{x}_{\mathrm{h}}^{T} \dot{x}_{\mathrm{h}}+I_{\mathrm{h}} \dot{\theta}_{\mathrm{h}}^{2}+m_{\mathrm{o}} \dot{x}_{\mathrm{o}}^{T} \dot{x}_{\mathrm{o}}+I_{\mathrm{o}} \dot{\theta}_{\mathrm{o}}^{2}\right), \\
U & =g\left(m_{\mathrm{h}} x_{\mathrm{h}}^{T}+m_{\mathrm{o}} x_{\mathrm{o}}^{T}\right)\left[\begin{array}{l}
0 \\
1
\end{array}\right]
\end{aligned}
$$

where $m_{\mathrm{h}}, I_{\mathrm{h}}$ and $m_{\mathrm{o}}, I_{\mathrm{o}}$ are the mass and moment of inertia of the hand and object, respectively, and $g$ is the gravitational acceleration. Inserting the expressions (4), (7), and (8) into Lagrange's equations (9) yields the dynamic equations. 


\section{Equations for the Disk-on-Disk System}

We now derive the specific equations for the disk-on-disk shown in Fig. 2. The radii of the hand and object disks are $r_{\mathrm{h}}$ and $r_{\mathrm{o}}$, respectively, and the hand is constrained to rotation only, i.e., $x_{\mathrm{h}}=0$.

Given a contact point expressed in the $u_{\mathrm{h}}-v_{\mathrm{h}}$ and the $u_{\mathrm{o}}-v_{\mathrm{o}}$ frames respectively as

$$
c_{\mathrm{h}}\left(s_{\mathrm{h}}\right)=\left[\begin{array}{c}
-r_{\mathrm{h}} \sin \left(s_{\mathrm{h}} / r_{\mathrm{h}}\right) \\
r_{\mathrm{h}} \cos \left(s_{\mathrm{h}} / r_{\mathrm{h}}\right)
\end{array}\right], c_{\mathrm{o}}\left(s_{\mathrm{h}}\right)=\left[\begin{array}{l}
-r_{\mathrm{o}} \sin \left(s_{\mathrm{h}} / r_{\mathrm{o}}\right) \\
-r_{\mathrm{o}} \cos \left(s_{\mathrm{h}} / r_{\mathrm{o}}\right)
\end{array}\right],
$$

from (1) and (2) we obtain

$$
\kappa_{\mathrm{h}}=1 / r_{\mathrm{h}}, \kappa_{\mathrm{o}}=-1 / r_{\mathrm{o}}, \kappa_{\mathrm{r}}=\frac{r_{\mathrm{h}}+r_{\mathrm{o}}}{r_{\mathrm{h}} r_{\mathrm{o}}} .
$$

Consequently, using (3)-(5), we obtain

$$
\theta_{\mathrm{o}}=\theta_{\mathrm{h}}+\kappa_{\mathrm{r}} s_{\mathrm{h}}, \dot{\theta}_{\mathrm{o}}=\dot{\theta}_{\mathrm{h}}+\kappa_{\mathrm{r}} \dot{s}_{\mathrm{h}}, \ddot{\theta}_{\mathrm{o}}=\ddot{\theta}_{\mathrm{h}}+\kappa_{\mathrm{r}} \ddot{\mathrm{s}}_{\mathrm{h}} .
$$

Substituting (12) into (7) and (8) and rearranging, we have

$$
\begin{aligned}
x_{\mathrm{o}} & =\left[\begin{array}{c}
-\left(r_{\mathrm{h}}+r_{\mathrm{o}}\right) \sin \left(\theta_{\mathrm{h}}+s_{\mathrm{h}} / r_{\mathrm{h}}\right) \\
\left(r_{\mathrm{h}}+r_{\mathrm{o}}\right) \cos \left(\theta_{\mathrm{h}}+s_{\mathrm{h}} / r_{\mathrm{h}}\right)
\end{array}\right], \\
\dot{x}_{\mathrm{o}} & =\left[\begin{array}{c}
-\left(r_{\mathrm{h}}+r_{\mathrm{o}}\right) \cos \left(\theta_{\mathrm{h}}+s_{\mathrm{h}} / r_{\mathrm{h}}\right)\left(\dot{\theta}_{\mathrm{h}}+\dot{s}_{\mathrm{h}} / r_{\mathrm{h}}\right) \\
-\left(r_{\mathrm{h}}+r_{\mathrm{o}}\right) \sin \left(\theta_{\mathrm{h}}+s_{\mathrm{h}} / r_{\mathrm{h}}\right)\left(\dot{\theta}_{\mathrm{h}}+\dot{s}_{\mathrm{h}} / r_{\mathrm{h}}\right)
\end{array}\right] .
\end{aligned}
$$

Inserting these expressions into Lagrange's equations, we solve for the dynamics

$$
\begin{aligned}
& m_{11} \ddot{\theta}_{\mathrm{h}}+m_{12} \ddot{s}_{\mathrm{h}}+h_{1}(q)=\tau_{\mathrm{h}}, \\
& m_{21} \ddot{\theta}_{\mathrm{h}}+m_{22} \ddot{s}_{\mathrm{h}}+h_{2}(q)=0,
\end{aligned}
$$

where $m_{11}=I_{\mathrm{h}}+I_{\mathrm{o}}+m_{\mathrm{o}}\left(r_{\mathrm{h}}+r_{\mathrm{o}}\right)^{2}, m_{12}=m_{21}=m_{\mathrm{o}}\left(r_{\mathrm{h}}+\right.$ $\left.r_{\mathrm{o}}\right)^{2} / r_{\mathrm{h}}+I_{\mathrm{o}} \kappa_{\mathrm{r}}, m_{22}=\kappa_{\mathrm{r}}^{2}\left(m_{\mathrm{o}} r_{\mathrm{o}}^{2}+I_{\mathrm{o}}\right), h_{1}(q)=-m_{\mathrm{o}} g\left(r_{\mathrm{h}}+\right.$ $\left.r_{\mathrm{o}}\right) \sin \left(\theta_{\mathrm{h}}+s_{\mathrm{h}} / r_{\mathrm{h}}\right)$, and $h_{2}(q)=-m_{\mathrm{o}} g r_{\mathrm{o}} \kappa_{\mathrm{r}} \sin \left(\theta_{\mathrm{h}}+s_{\mathrm{h}} / r_{\mathrm{h}}\right)$.

In our experimental implementation, we use a highlygeared harmonic drive DC motor to drive the hand. For this reason, it is more convenient to consider the hand's angular acceleration (created by a low-level acceleration controller) as the system input rather than the torque $\tau_{\mathrm{h}}$ in (17a). Defining this new acceleration control as $v=\ddot{\theta}_{\mathrm{h}}$ and substituting into (17), we rewrite the dynamics as

$$
\begin{aligned}
& \ddot{\theta}_{\mathrm{h}}=v, \\
& \ddot{s}_{\mathrm{h}}=-\frac{1}{m_{22}}\left(m_{12} v+h_{2}(q)\right) .
\end{aligned}
$$

By plugging (18) into (17a), the relationship between the hand torque $\tau_{\mathrm{h}}$ and the new input $v$ is found to be

$$
\tau_{\mathrm{h}}=\left(m_{11}-\frac{m_{12}^{2}}{m_{22}}\right) v-\frac{m_{12}}{m_{22}} h_{2}+h_{1} .
$$

\section{Friction Constraints}

The previous derivations are predicated on the assumption that $s_{\mathrm{h}}=s_{\mathrm{o}}$ at all times, i.e., rolling is guaranteed. For some states and controls, however, rolling requires the hand to "pull" on the object (negative normal force $f_{\mathrm{n}}$ ), and in others the required friction force magnitude $f_{\mathrm{f}}$ may be greater than $\mu f_{\mathrm{n}}$, where $\mu$ is the Coulomb friction coefficient. The condition that the contact forces satisfy the contact friction constraint can be expressed abstractly as a constraint on the state $(q, \dot{q})$ and control $v$ :

$$
0 \leq F(q, \dot{q}, v) \leq \mu .
$$

The larger the friction coefficient $\mu$, the larger the volume of the state-control space that satisfies the friction conditions for rolling. Outside of this volume, the object slips or falls from the hand.

\section{STABILIZATION CONTROL}

In this section we propose a stabilizing controller based on backstepping.

The system described by (18) can be expressed in statespace form with the state variable $z=\left[\theta_{\mathrm{h}}, \dot{\theta}_{\mathrm{h}}, s_{\mathrm{h}}, \dot{s}_{\mathrm{h}}\right]^{T}$ as

$$
\begin{aligned}
& \dot{z}_{1}=z_{2}, \\
& \dot{z}_{2}=v, \\
& \dot{z}_{3}=z_{4}, \\
& \dot{z}_{4}=-\frac{h_{2}(z)}{m_{22}}-\frac{m_{12}}{m_{22}} v .
\end{aligned}
$$

The job of the controller is to drive both velocities $z_{2}$ and $z_{4}$ to zero with the center of mass of the object directly above the center of the hand (the balanced position). It is not possible to independently control both $z_{1}$ and $z_{3}$, however; they couple to determine the location of the center of mass of the object. For this reason, we project away one of the state dimensions and introduce the following change of coordinates:

$$
\begin{aligned}
\eta_{1} & =m_{22} \dot{s}_{\mathrm{h}}+m_{12} \dot{\theta}_{\mathrm{h}}, \\
\eta_{2} & =\theta_{\mathrm{h}}+s_{\mathrm{h}} / r_{\mathrm{h}}, \\
\xi & =\dot{\theta}_{\mathrm{h}} .
\end{aligned}
$$

The new state $\eta_{2}$ represents the angular position of the center of the object measured counterclockwise with respect to the $y$-axis of the world frame. This is the only configuration we are concerned with. The states $\eta_{1}$ and $\xi$ span the original velocity space $z_{2}-z_{4}$. The reduced dynamics can now be written

$$
\begin{aligned}
& \dot{\eta}_{1}=\underbrace{\frac{m_{\mathrm{o}} g\left(r_{\mathrm{h}}+r_{\mathrm{o}}\right)}{r_{\mathrm{h}}}}_{\equiv \sigma_{1}} \sin \left(\eta_{2}\right), \\
& \dot{\eta}_{2}=\underbrace{\frac{1}{r_{\mathrm{h}} m_{22}}}_{\equiv \sigma_{2}} \eta_{1}+\underbrace{\left(1-\frac{m_{12}}{r_{\mathrm{h}} m_{22}}\right)}_{\equiv \sigma_{3}} \xi, \\
& \dot{\xi}=v .
\end{aligned}
$$

We now use the following two theorems to apply backstepping to the governing equations (22).

Theorem 3.1 ([10], [11]): Consider the system

$$
\begin{aligned}
& \dot{z}=f(z, \xi) \\
& \dot{\xi}=u
\end{aligned}
$$

where $z \in \mathbb{R}^{n}, \xi \in \mathbb{R}$, and $f$ is a smooth function satisfying $f(0,0)=0$. Assume that the subsystem (23a) is stabilized by 
a smooth state feedback control law $\xi=\alpha(z)$ with $\alpha(0)=$ 0 and let $V(z)$ be a smooth, positive-definite, and radially unbounded Lyapunov function satisfying

$$
\frac{\partial V}{\partial z} f(z, \alpha(z))<0, \quad \forall z \neq 0 .
$$

Then the following state feedback control law

$$
u=\frac{\partial \alpha}{\partial z} f(z, \xi)-\frac{\partial V}{\partial z} h(z, \mu)-c \mu
$$

globally asymptotically stabilizes the origin of the overall system (23), where $c>0, \mu=\xi-\alpha(z)$, and

$$
h(z, \mu):=\int_{0}^{1}\left[\frac{\partial f(z, \zeta)}{\partial \zeta}\right]_{\zeta=\alpha(z)+s \mu} d s .
$$

Proof: The result follows from the Lyapunov function $W(z, \xi)=V(z)+\frac{1}{2} \mu^{2}$. See [10] or [11].

Theorem 3.2 ([11], [12]): Consider the nonlinear system

$$
\begin{aligned}
& \dot{z}=f(z)+g(z) \xi \\
& \dot{\xi}=u
\end{aligned}
$$

where $z \in \mathbb{R}^{n}$, and $f, g$ are smooth functions satisfying $f(0)=0$. Let the state feedback $\xi=\alpha(z)$ satisfying $\alpha(0)=$ 0 globally asymptotically stabilize $z=0$. Then the following smooth state feedback

$$
u=\frac{\partial \alpha}{\partial z}(f(z)+g(z) \xi)-\frac{\partial V}{\partial z} g(z)-c(\xi-\alpha(z))
$$

globally asymptotically stabilizes $(z, \xi)=0$ for the overall system (25).

Proof: See [11] or [12].

Now we propose a globally asymptotically stabilizing controller for the disk-on-disk system (22).

Proposition 3.3: The subsystem consisting of (22a) and (22b) is globally asymptotically stabilized to $\left(\eta_{1}, \eta_{2}\right)=$ $(0,0)$ with the state feedback $\xi=\alpha_{1}\left(\eta_{1}, \eta_{2}\right)$, where

$$
\alpha_{1}=\frac{1}{\sigma_{3}}\left\{\frac{\partial \alpha}{\partial \eta_{1}}\left[\sigma_{1} \sin \left(\eta_{2}\right)\right]-\frac{\partial V}{\partial \eta_{1}} h(\eta, \mu)-c \mu-\sigma_{2} \eta_{1}\right\},
$$

where $\alpha\left(\eta_{1}\right)=-c_{0} \tanh \left(c_{1} \eta_{1}\right)$ with $0<c_{0}<\frac{\pi}{2}$ and $c_{1}>0, V\left(\eta_{1}\right)=\frac{1}{2} \eta_{1}^{2}, \mu=\eta_{2}-\alpha\left(\eta_{1}\right), c>0$, and $h=\int_{0}^{1} \sigma_{1} \cos \left(\alpha\left(\eta_{1}\right)+s \mu\right) d s$ as defined in Theorem 3.1.

Furthermore, the overall system (22) is globally asymptotically stabilized to the origin $\left(\eta_{1}, \eta_{2}, \xi\right)=(0,0,0)$ with the state feedback

$$
v=\frac{\partial \alpha_{1}}{\partial \eta}[f(\eta)+g(\eta) \xi]-\frac{\partial W}{\partial \eta} g(\eta)-k\left(\xi-\alpha_{1}(\eta)\right),
$$

where $W\left(\eta_{1}, \eta_{2}\right)=V\left(\eta_{1}\right)+\frac{1}{2} \mu^{2}, k>0$, and $f(\eta), g(\eta)$ are given by the $\eta$-subsystem in affine form such that

$$
\dot{\eta}=f(\eta)+g(\eta) \xi=\left[\begin{array}{c}
\sigma_{1} \sin \left(\eta_{2}\right) \\
\sigma_{2} \eta_{1}
\end{array}\right]+\left[\begin{array}{c}
0 \\
\sigma_{3}
\end{array}\right] \xi .
$$

Proof: First, the state feedback $\eta_{2}=\alpha\left(\eta_{1}\right)$ globally asymptotically stabilizes the subsystem (22a) with a Lyapunov function $V\left(\eta_{1}\right)=\frac{1}{2} \eta_{1}^{2}$. Next, the input transformation $\xi=\frac{1}{\sigma_{3}}\left(u-\sigma_{2} \eta_{1}\right)$ reduces (22b) to $\dot{\eta}_{2}=u$. Now applying Theorem 3.1 for this $\eta$-subsystem yields the state feedback
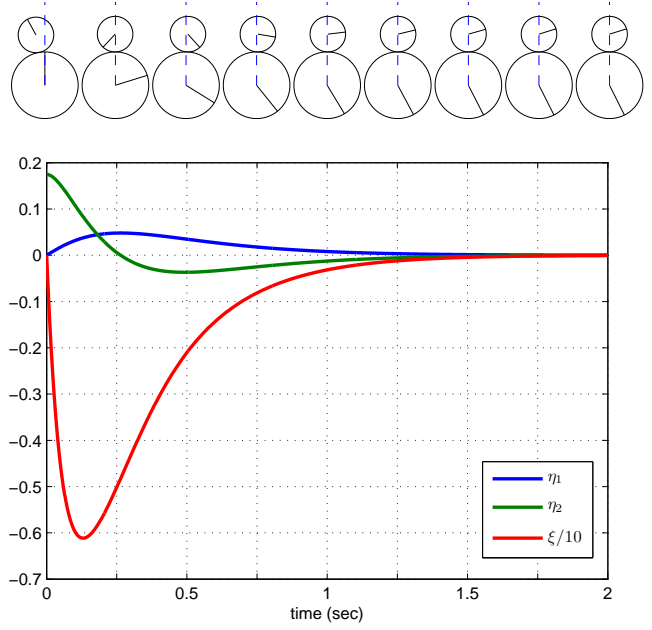

Fig. 3. Simulation results showing $\eta_{1}, \eta_{2}$, and $\xi$ converging to zero. The quantity $\frac{1}{10} \xi$ is plotted instead of $\xi$ so it can be shown on the same scale as $\eta_{1}$ and $\eta_{2}$. The icons at top show the configurations of the disks every 0.25 seconds.

$\xi=\alpha_{1}\left(\eta_{1}, \eta_{2}\right)$ with a Lyapunov function $W\left(\eta_{1}, \eta_{2}\right)=$ $V\left(\eta_{1}\right)+\frac{1}{2} \mu^{2}$.

We next apply Theorem 3.2, the affine version of Theorem 3.1 , to the overall system (22) in which the $\eta$-subsystem is in affine form $\dot{\eta}=f(\eta)+g(\eta) \xi$. This yields the globally asymptotically stabilizing state feedback $v$ for the overall system (22). The associated Lyapunov function for the overall system is given by $\bar{W}=W\left(\eta_{1}, \eta_{2}\right)+\frac{1}{2}\left(\xi-\alpha_{1}\right)^{2}$.

We conclude by noting that the dynamics of the transformed disk-on-disk system (22) have the same form as the dynamics of the inertia wheel pendulum [13], which consists of a free-swinging pendulum with an actuated wheel at the free end. Olfati-Saber presents an alternative control law for the inertia wheel pendulum in [11].

\section{Simulation Results}

Simulation results with the control law (28) are shown in Figs. 3-4. The values of parameters used in the simulation are the actual values of the disk-on-disk system constructed for experiment: $m_{\mathrm{o}}=0.142 \mathrm{~kg}, r_{\mathrm{o}}=0.08 \mathrm{~m}, I_{\mathrm{o}}=0.4544 \times$ $10^{-3} \mathrm{~kg} \cdot \mathrm{m}^{2}, r_{\mathrm{h}}=0.15 \mathrm{~m}$, and $I_{\mathrm{h}}=5.36 \times 10^{-3} \mathrm{~kg} \cdot \mathrm{m}^{2}$. All angles are measured in radians, and all units are SI unless otherwise noted.

According to Proposition 3.3, global asymptotic stability is guaranteed for positive gains $\left(c_{0}, c_{1}, c, k\right)$ with $c_{0}<\pi / 2$. In practice, because the control law is nonlinear, gains yielding good transient performance are found experimentally. We chose $\left(c_{0}, c_{1}, c, k\right)=(1.5,5,4,4)$ for the simulation. Given the initial condition $\eta_{2}(0)=0.175$ and assuming $\theta_{\mathrm{h}}(0)=$ 0 , the corresponding initial conditions of $\theta_{\mathrm{o}}$ and $s_{\mathrm{h}}$ are calculated as $\theta_{\mathrm{o}}(0)=0.502$ and $s_{\mathrm{h}}(0)=0.026$ from (14) and (21b).

In the simulation, as shown in Fig. 3, all state variables $\eta_{1}$, $\eta_{2}$, and $\xi$ converge to zero, which physically means that the object stabilizes to the position directly above the hand and that the angular velocities of the hand and object converge to zero. The icons above Fig. 3 show the configurations of 


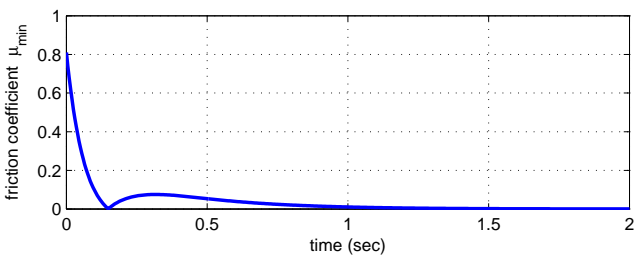

Fig. 4. A plot of the minimum friction coefficient required to prevent slipping at the contact during stabilization. The friction force changes sign at approximately $t=0.15 \mathrm{~s}$.

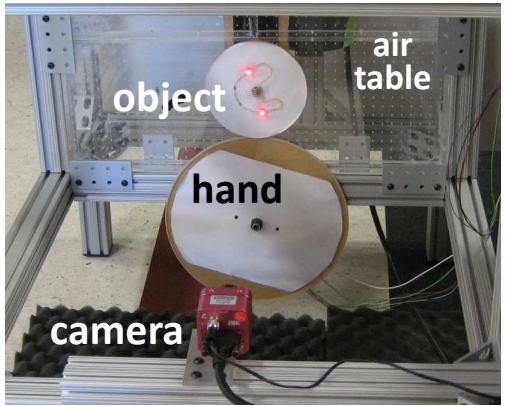

Fig. 5. The disk-on-disk experimental setup. The hand is actuated by a harmonic drive DC motor. A high speed camera and vision system is used for visual feedback of the object.

the two disks every 0.25 seconds. Note that the orientation of the hand does not converge to zero while the velocity of the hand $\xi$ goes asymptotically to zero. This is because, as mentioned earlier, we do not directly control $\theta_{\mathrm{h}}$ or $s_{\mathrm{h}}$ individually, thus those variables are not necessarily required to converge to zero. Figure 4 plots the minimum friction coefficient $\mu$ needed to ensure rolling as a function of time. At the beginning of the run, when the object is left of center, the large control acceleration $v$ requires a large friction coefficient to prevent slipping. As the object becomes balanced above the hand, the friction coefficient required for rolling drops to zero.

\section{EXPERIMENTAL RESULTS}

\section{A. Experimental Setup}

Figure 5 shows our disk-on-disk experimental setup. The hand is the lower disk, actuated by a harmonic drive DC motor (Harmonic Drive RH-8D 6006) equipped with a 50:1 gear box and a 500 ppr quadrature encoder giving 100,000 counts per revolution at the output shaft. The object disk is free to roll on the hand. The disk-on-disk system is mounted on an air table that provides frictionless support in a plane tilted $85.2^{\circ}$ with respect to horizontal (99.6\% of full gravity). Both hand and object are made of $1 / 4$ inch thick acrylic and the hand is encircled by a rubber band to increase friction.

The hand control algorithm runs on a PC104 stack with a QNX real-time operating system (RTOS). In addition to the angular position data from the encoder of the hand, the controller utilizes the state of the object disk, which is determined using a high-speed vision system. The vision system, consisting of a PhotonFocus TrackCam camera, a Microenable III frame grabber, and a Windows PC, allows us to implement real-time control of the system at $800 \mathrm{~Hz}$. The vision program, written in $\mathrm{C}++$, tracks two dots (LEDs)

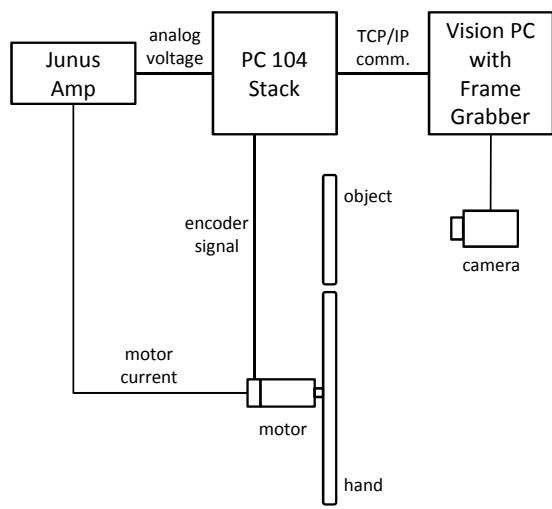

Fig. 6. Schematic of the experimental set-up showing signal paths with a side view of the hand and object. The actual disk-on-disk system is tilted $85.2^{\circ}$ with respect to the horizontal plane.

on the object, allowing us to calculate its position and orientation. This information is sent to the controller PC104 stack via TCP/IP.

\section{B. Controller Implementation}

The control algorithm is written in $\mathrm{C}++$ and runs on the PC104 stack. Using the QNX RTOS, the control loop runs at $800 \mathrm{~Hz}$ in sync with the vision system. As the control law requires the angular velocity of each disk, we apply differencing plus low-pass filters to the encoder and vision data to estimate velocity.

Since our control law (28) generates acceleration commands $v$, we implement an additional acceleration control loop converting these controls to the motor current requested from the motor amplifier (Copley Controls Junus 800-1468). This inner controller is written

$$
i_{\text {com }}=i_{\mathrm{ff}}\left(\dot{\theta}_{\mathrm{h}}, v\right)+k_{\mathrm{p}}\left(\theta_{\text {ref }}-\theta_{\mathrm{h}}\right)+k_{\mathrm{d}}\left(\dot{\theta}_{\text {ref }}-\dot{\theta}_{\mathrm{h}}\right),
$$

where $i_{\text {com }}$ is the commanded motor current, $i_{\mathrm{ff}}$ is a feedforward motor current based on the hand's angular velocity and requested acceleration, $\theta_{\text {ref }}$ and $\dot{\theta}_{\text {ref }}$ are the desired hand position and velocity obtained by integrating the acceleration command $v(t)$, and $k_{\mathrm{p}}$ and $k_{\mathrm{d}}$ are PD gains. The feedforward motor current is based on the simple model

$$
\left(J_{\mathrm{L}}+J_{\mathrm{m}}\right) \ddot{\theta}_{\mathrm{h}}=k_{\mathrm{m}} i-\mu_{\mathrm{d}} \dot{\theta}_{\mathrm{h}}-f_{\mathrm{s}} \operatorname{sgn}\left(\dot{\theta}_{\mathrm{h}}\right),
$$

where $J_{\mathrm{L}}$ and $J_{\mathrm{m}}$ denote the inertia of the hand and the motor rotor, respectively, $k_{\mathrm{m}}$ is the motor constant, $i$ is the motor current, $\mu_{\mathrm{d}}$ is a viscous friction coefficient, $f_{\mathrm{s}}$ is the torque required to overcome friction from rest, and the function $\operatorname{sgn}(\cdot)$ returns the sign of its argument. The inertia of the motor $J_{\mathrm{m}}$ is the reflected inertia due to the gear box. We estimated the values of $k_{\mathrm{m}}, \mu_{\mathrm{d}}$, and $f_{\mathrm{s}}$ in motor modeling experiments, and the feedforward current $i_{\mathrm{ff}}$ is calculated by substituting the commanded acceleration $v$ and current hand velocity $\dot{\theta}_{\mathrm{h}}$ into (30). After estimating the parameters of the feedforward model, the PD gains were tuned experimentally to reject modeling errors, yielding $\left(k_{\mathrm{p}}, k_{\mathrm{d}}\right)=(150,0.9)$.

\section{Results and Discussion}

Figure 7 shows the state variables $\left(\eta_{1}, \eta_{2}, \xi\right)$ for one experimental run. The initial condition of the experiment was 

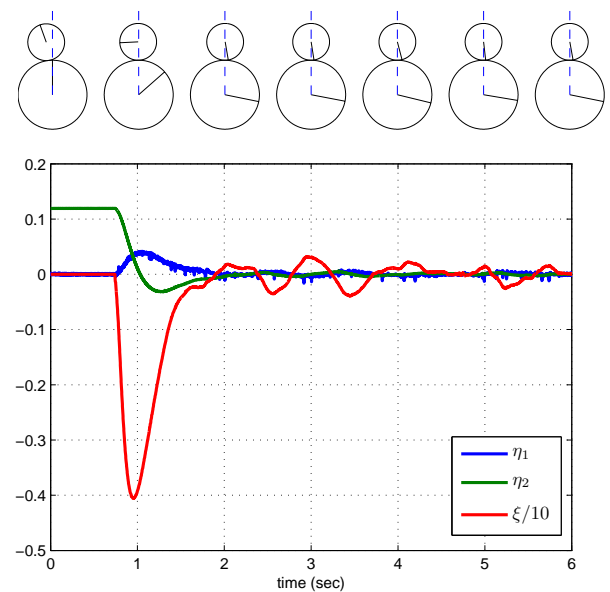

Fig. 7. Experimental result showing the state variables $\eta_{1}, \eta_{2}, \xi$. The control action starts at $t=0.75 \mathrm{~s}$.

set at $\left(\eta_{1}, \eta_{2}, \xi\right)=(0,0.119,0)$, which means that initially both disks were at rest and the object disk was placed with its center of mass at an angle of $0.119 \mathrm{rad}\left(6.82^{\circ}\right)$ relative to the world $y$-axis. The object disk was kept at rest at the initial position using a small block until control action starts at $t=0.75 \mathrm{~s}$. After $\eta_{2}$ settles within the range of $\pm 5 \%$ of the initial condition, i.e., $0.006 \mathrm{rad}$, at $t=1.93 \mathrm{~s}$, the RMS error of $\eta_{2}$ is $0.0018\left(0.103^{\circ}\right)$. Similarly, after $t=1.93 \mathrm{~s}$, the RMS errors of $\eta_{1}$ and $\xi$, which are related to the angular velocities of the object and hand, are 0.0024 and 0.1438 , respectively. Figure 8 shows that the inner loop acceleration controller (29) provides satisfactory tracking of the commanded hand acceleration $v$.

The control gains used in our experiments are $\left(c_{0}, c_{1}, c, k\right)=(0.6,5.0,3.5,3.5)$, which are similar but not identical to our simulation gains. The simulation and experiment differ in a number of ways, including discrete-time implementation, approximate control of the motor acceleration, quantized position sensing, continuous small perturbations from the air table, etc. We found that these experimental gains reduced the appearance of small-amplitude limit cycles near the balanced configuration.

The friction coefficient to prevent slipping is required to be greater than 0.31 for this experiment, which is the largest at the beginning of the run. The actual friction coefficient of 2.0, which is obtained from an empirical measurement, ensures rolling during the experiment.

We might ask whether it is possible to balance the object at a position not directly above the center of the hand. This is not practically possible, as the vertical contact force needed to cancel gravity necessarily creates a moment about the object's center of mass. This means that the object (and the hand) must rotate at a constant angular acceleration, resulting in unbounded velocities. This can be seen in equations (22) by plugging a constant value into $\eta_{2}$ and setting $\dot{\eta}_{2}=0$.

We are currently working on another valid control objective of stabilization at the upright position while both the hand and object disks rotate (roll) at constant velocities or rotate to a specific orientation.

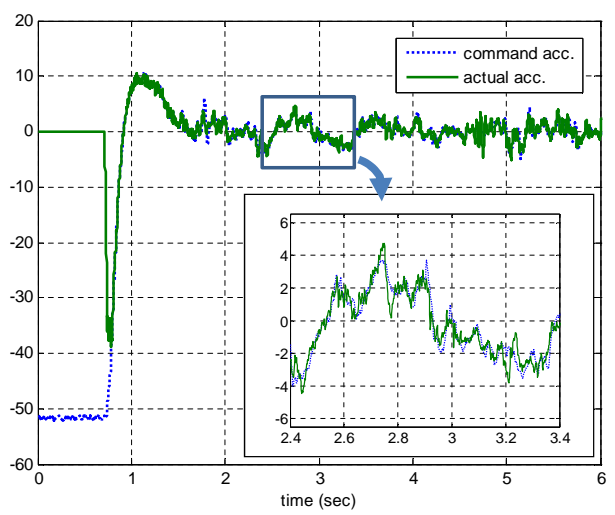

Fig. 8. Experimental result showing the command and actual accelerations. The actual acceleration follows the command acceleration satisfactorily.

\section{CONCLUSIONS}

In this paper, we derived the kinematic and dynamic equations of the disk-on-disk system. Based on these, we derived and demonstrated a controller to balance the object disk at the unstable upright position. Future work will focus on motion planning and feedback stabilization of a broad class of rolling trajectories for smooth planar objects rolling on smooth planar hands (other than disks) moving with a full three degrees-of-freedom.

\section{ACKNOWLEDGMENTS}

We thank Philip Dames for his help with the construction of the experimental setup, Nelson Rosa for writing the vision code, and Georg Bätz for helpful discussions. This work was supported by NSF grant IIS-0964665.

\section{REFERENCES}

[1] K. M. Lynch and M. T. Mason, "Dynamic underactuated nonprehensile manipulation," in Proc. IEEE/RSJ Int. Conf. on Intelligent Robots and Systems (IROS), 1996, pp. 889-896.

[2] — , "Dynamic nonprehensile manipulation: Controllability, planning, and experiments," Int. J. of Robotics Research, vol. 18, no. 1, pp. 64-92, 1999.

[3] K. M. Lynch, N. Shiroma, H. Arai, and K. Tanie, "The roles of shape and motion in dynamic manipulation: The butterfly example," in Proc. IEEE Int. Conf. on Robotics and Automation, 1998, pp. 1958-1963.

[4] J. Hauser, S. Sastry, and P. Kokotovic, "Nonlinear control via approximate input-output linearization: the ball and beam example," IEEE Transactions on Automatic Control, vol. 37, pp. 392-398, 1992.

[5] C. Barbu, R. Sepulchre, W. Lin, and P. V. Kokotovic, "Global asymptotic stabilization of the ball-and-beam system," in Proc. IEEE Conf. on Decision and Control, vol. 3, 1997, pp. 2351-2355.

[6] F. Borrelli, "Ball and plate," in Constrained Optimal Control of Linear and Hybrid Systems (Lecture Notes in Control and Information Sciences), vol. 290. Springer, 2003, pp. 177-183.

[7] G. Oriolo and M. Vendittelli, "A framework for the stabilization of general nonholonomic systems with an application to the plate-ball mechanism," IEEE Transactions on Robotics, vol. 21, pp. 162-175, 2005.

[8] X. Yun, V. Kumar, N. Sarkar, and E. Paljug, "Control of multiple arms with rolling constraints," in Proc. IEEE Int. Conf. on Robotics and Automation, 1992, pp. 2193-2198.

[9] D. J. Montana, "The kinematics of contact and grasp," Int. J. of Robotics Research, vol. 7, pp. 17-32, 1988.

[10] A. Isidori, Nonlinear Control Systems, 3rd ed. Springer, 1995.

[11] R. Olfati-Saber, "Nonlinear control of underactuated mechanical systems with application to robotics and aerospace vehicles," Ph.D. dissertation, Massachusetts Institute of Technology, 2001.

[12] H. K. Khalil, Nonlinear Systems, 3rd ed. Prentice Hall, 2001.

[13] M. W. Spong, P. Corke, and R. Lozano, "Nonlinear control of the inertia wheel pendulum," Automatica, vol. 37, pp. 1845-1851, 1999. 